\title{
STABILITY PROPERTIES OF THE GENERALIZED CHERNOFF INEQUALITY
}

\section{GAO AND YILING WANG}

Abstract. In this short note we will present two stability properties of the Chernoff-Ou-Pan inequality, newly obtained in [6], which states that if $K$ is a convex domain in the plane $\mathbb{R}^{2}$ with area $a(K)$, then one gets

$$
a(K) \leqslant \frac{1}{k} \int_{0}^{\frac{\pi}{k}} \omega_{k}(\theta) w_{k}\left(\theta+\frac{\pi}{k}\right) d \theta,
$$

where $w_{k}(\theta)$ is defined in [6] (see also $\S 3$ below), and the equality holds if and only if $K$ is a circular disc.

Mathematics subject classification (2010): 52A38,52A40.

Keywords and phrases: Stability, convex domains, Chernoff-Ou-Pan inequality, Fourier series.

\section{REFERENCES}

[1] P. R. Chernoff, An area-width inequality for convex curves, Amer. Math. Monthly 76, 1 (1969), $34-35$.

[2] B. Fuglede, Stability in the isoperimetric problem, Bull. London Math. Soc. 18 (1986), 599-605.

[3] H. Groemer, Stability properties of geometric inequalities, Amer. Math. Monthly 97 (1990), 382 394.

[4] H. Groemer, Stability properties of geometric inequalities, In: Handbook of Convex Geometry, P.M. Gruber and J.M. Wills (eds), North Holland, 125-150, 1993.

[5] C. C. Hsiung, , A First Course in Differential Geometry, Pure \& Applied Math., Wiley, New York, 1981.

[6] K. OU \& S. L. PAN Some remarks about closed convex curves, Pacific J. Math., 248 (2010), 393-401.

[7] S. L. PAn \& H. P. XU, Stability of a reverse isoperimetric inequality, J. Math. Anal. Appl. 350 (2009), $348-353$ 